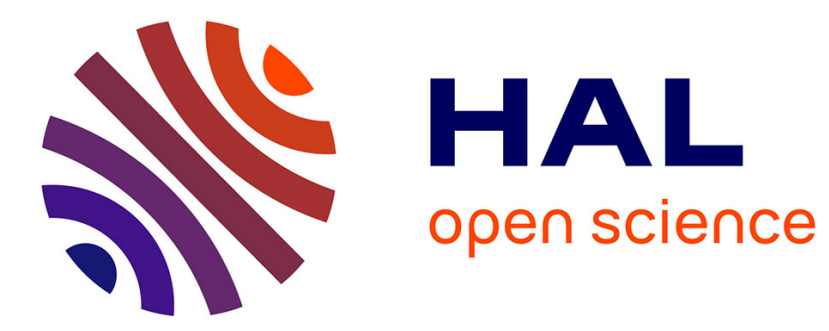

\title{
Can Component/Service-Based Systems Be Proved Correct?
}

\author{
Christian Attiogbe
}

\section{To cite this version:}

Christian Attiogbe. Can Component/Service-Based Systems Be Proved Correct?. Conference on Current Trends in Theory and Practice of Computer Science, Czech Republic, Jan 2009, Spindleruv Mlýn,, Czech Republic. pp.3-18, 10.1007/978-3-540-95891-8_2 . hal-00420051

\section{HAL Id: hal-00420051 https://hal.science/hal-00420051}

Submitted on 9 Oct 2009

HAL is a multi-disciplinary open access archive for the deposit and dissemination of scientific research documents, whether they are published or not. The documents may come from teaching and research institutions in France or abroad, or from public or private research centers.
L'archive ouverte pluridisciplinaire HAL, est destinée au dépôt et à la diffusion de documents scientifiques de niveau recherche, publiés ou non, émanant des établissements d'enseignement et de recherche français ou étrangers, des laboratoires publics ou privés. 


\title{
Can Component/Service-based Systems be Proved Correct?
}

\author{
J. Christian Attiogbé \\ LINA - UMR CNRS 6241 - University of Nantes - France \\ Christian.Attiogbe@univ-nantes.fr
}

(Technial Report version)

\begin{abstract}
Component-oriented and service-oriented approaches have gained a strong enthusiasm in industries and academia with a particular interest for service-oriented approaches. The service concept and its use in web-based application development have a huge impact on reuse practices. Accordingly a considerable part of software architectures is influenced; these architectures are moving towards service-oriented architectures. Therefore applications (re)use services that are available elsewhere and many applications interact, without knowing each other, using services available via service servers and through their published interfaces and functionalities.

Industries propose, through various consortium, languages, technologies and standards. More academical works are also undertaken concerning semantics and formalisation of components and service-based systems. We consider here both streams of works in order to raise research concerns that will help in building quality software.

Are there new challenging problems with respect to service-based software construction.
\end{abstract}

\section{Introduction}

Component-oriented and service-oriented approaches have gained a strong enthusiasm in industries and academia with a particular interest for service-oriented ones.

A component is a software entity with given functionalities, made available by a provider, and used to build other application within which it is integrated. The concept of service as a specific software entity which is delivered upon request via a network is not a new idea; think about telecommunication services, IP network services, etc.

A service is a software entity that is designed for a given functionality, is made available through a network via a provider, and is linked and used on demand. An example is a calendar service used within a flight booking application in order to help the user in defining its flight departing and returning dates. The calendar service is a service integrated in the booking application; it may be replaced by another one or may even be disconnected, in which case the user may for example enter manually her/his dates. 
The service concept and its use in web-based application development have a huge impact on reuse practices. Accordingly a considerable part of software architectures is influenced; these architectures are moving towards service-oriented architectures (SOA). Applications (re)use services that are available elsewhere and several applications interact, without knowing each other, using the services available via service servers and their published interfaces and functionalities.

The application designers are confident in the used services and their (unknown) providers; they are also confident in the used communication protocols, even if the services may have misleading semantics. Important issues at this stage are the description, the storage, the management and the retrieval of appropriate services from large certified libraries. Standardisation effort is therefore unavoidable to ensure certification.

Software service descriptions take various forms; services are described either inside a software component which role is to provide a computation, or inside a software component which provides several other services as functionalities; services may be described using software components.

When an external service is considered within a more general software application, one should think about the meaning of the interaction between the application and the external service and, consequently, the meaning of the application with or without the links with these external services. It is worth mentioning a few key features: service description, service discovery, service interaction, service-oriented applications and their architectures. In [15] Fiadeiro advocates for the emerging of a new discipline concerning software services. Papazoglou and van den Heuvel propose a general panel in 25] of the technology around service-oriented architecture. An accompanying work can be found in [24].

Are there new challenging problems with respect to service-based software construction, to service construction, to software verification? Besides, what are the links and the advances compared to distributed systems? Do the technical proposals meet the industry expectations? what are the current trends and the perspectives of service-oriented approaches?

Considering the ubiquitous aspect of large scale distributed applications, the way software services are impacting software construction or the way they are being used or researched requires special care. The standardisation of service description languages constitutes an important step but is not yet sufficient. The meaning of services, their constraints and their properties are unavoidable to guarantee the well-functioning of global distributed applications that use them.

The main message stressed here, is that there is a real need to think about services and components as software building units that should last several years or decades and that require for this purpose technical specifications and the guarantee of well-functioning. Indeed today Information Systems and Applications, due to the Internet, are inter-penetrated in such a way that failures or defects of some components or services may drastically impact users and their activities in the future.

Then, a specific emphasis should be put on correctness properties of services and on service-based systems in order to ensure their quality. An important re- 
search issue is to reason on the correctness of software applications that will dynamically use or embed existing services provided by a third party. For example, additionally to the formal specification of its functionalities, a service may embed its specific properties and the certificate or the proof that guarantees these properties.

From the methodological point of view, any given " $\alpha$-oriented" development method requires a clear description of what $\alpha$ is or supposed to be; therefore helping to differentiate an $\alpha$-oriented approach from a $\beta$-oriented one.

An $\alpha$-oriented approach should make it explicit some concepts, laws, rules, guidance or method to apply it distinctly from $\beta$-oriented approach even if an $\alpha$-oriented approach may be based on $\beta$-oriented ones with respect to methodological purpose. From this point of view, it should be clear how to situate service-oriented development relatively to distributed-system development.

In the object-oriented approach for example, one considers the encapsulation of data and the methods that manipulate the data as elementary units (classes) which are related through inheritance, extension and client-ship.

From this point of view, service-oriented approaches require a clear positioning that is currently disseminated through various existing works. However a systematic view that will favour the emergence of mature seamless development process and integrated tools and also academic courses is recommendable.

According to a given business application a software functionality is a specific need which occurs time being, then an access and a link to the corresponding service is achieved on demand. The service should be first identified and the located before being used.

A current widely admitted practice of service-based approaches is that services are available somewhere in the Internet; they are accessed on request and a given application may perform, during its execution access to the available services. Therefore it is worth underlying the issue on service correctness and interoperability. All these are challenging concerns. The standardisation of service descriptions including their specifications (interfaces, behaviour and semantics), their properties and their quality is the only way to overcome these challenges.

The remaining of this article is structured around four sections. Section 2 is devoted to the introduction of the well-admitted notion of components and services. In Section 3 we deal with the analysis of component or services based systems. Section 4 is devoted to research issues and related directions. Finally in Section 5 we give some concluding remarks.

\section{Components and Services in Software Construction}

\subsection{Reuse of Software}

The reuse principle has always been present as a fundamental support for software construction. Functions, Modules and Classes have been the concrete entities that enforce reuse. In the same stream, Components and Services follow on, and are just the adaptation of the reuse practices to the environment of software construction. Indeed one may observe the evolution of the reuse entities 
from the point of view of centralised or decentralised environment. Functions, Modules and Classes are well-adapted to centralised environment. Libraries of these entities are available in situ for the software developers. With the maturity of network technologies and mainly the Internet protocols (via the TCP/IP facilities) the abstraction level of reuse entities changes: Component-based development appears in this context in the 90's. Clearly as the software technological environment changes, abstraction level of reuse entities changes. Services are now, the most widely highlighted reuse entities.

\subsection{Comparing Components and Services}

Components are viewed as the abstraction of a part or a subsystem of an application. The notion of component is widely used in many disciplines and industries. For example the Hardware in a computer is made of several linked components (the CPU, input-output devices, storage units, etc). A computer is made of several components that interact at different levels (within the Hardware, the operating system and in the applications).

Therefore a component is an independent building block that can be independently integrated in one application or within several applications. In a component-based approach the functionalities of a software are considered completely independent and designed as separate entities (the components) that can be composed afterthought in building a applications. The components can be designed and offered by a tier.

Services are software building blocks used in building distributed applications. They are also used to integrate existing functionalities into new applications, or used to make different distributed applications communicate. A service is provided by a service provider and required by a consumer or a service client; it can be dynamically located and invoked. Therefore the network of computers, more specifically the Internet, is the support and the vehicle of services and consequently one of the main characteristics of service-based systems.

Web-Services Web-services are a specific implementation of services; they are now very popular through the industrial offers. They use XML and the Internet TCP/IP popular protocol.

According to the W3O $A$ (web)service is a software system designed to support interoperable machine to machine interaction over a network.

Components and Services share many commonalities; they are both independent entities used as building blocks in the construction of software applications. They provide interfaces to enable usage and interactions. However, a component is a packaging unit of one or several services; therefore it provides one or several services. A service can be built using components but a service is not necessarily built from components. Services are clearly located at the functionalities abstraction level. Services correspond to offered functionalities.

\footnotetext{
1 The World Wide Web Consortium
} 
Components are known and assembled in order to enable the usage of services. Components are integrated and compiled within new applications that use them. They become a specific part of the new application. Services are not compiled; they are dynamically linked at runtime within the applications that use them.

Sharing aspect also particularise components and services. A priori a component is not shared between applications as the component is integrated within the application. One service may be shared by several clients. However there are variations on components and services that do not fit this distinction, components or their services may also be shared with specifically defined policies.

From the service-based approach, interactions are not statically predefined. The services do not know their caller; service clients and the called services are bound upon request where clients behave like if services are always available.

\subsection{Service-Based Systems}

A service-based system is one that (re)uses services available elsewhere in the Internet to achieve its own functionalities. Note that a service-based system may be partially or totally architectured with components. The components of the system request some functionalities that are described as services outside the system. According to a user, the system is completely independent and autonomous; the user does not see the interactions on the background network.

Service-based systems are distributed systems: they are systems made of collection of components (computers, software applications, devices, etc); they are perceived by a user as a single system. Their sub-systems are linked by a network equipped with communication protocols, and cooperate to share resources and to perform their assigned tasks. From the interaction point of view, services are facilities used by software applications to make communicate various sub-systems connected via the Internet in order to achieve given tasks.

Compared with classical distributed systems, service-based systems have the main features but are rather unpredictable for that their parts may be unknown at a given time. Indeed services are volatile distributed entities; they may be searched, dynamically linked with the rest of the system environment, and unlinked at another moment.

Web-Services based applications are service-based systems that use the Internet and its protocols as the distribution platform. They are the widely used specialisation of service-based systems.

\subsection{Event-based Style of Interaction}

An event-based style of communication is well-adapted to the construction of service-based systems. In an event-based style of interaction, the components of a distributed application communicate via events which are generated by ones components and received by others. This kind of interaction facilitates the decoupling of components. Indeed an event notification system or an event management middle-ware handles the interaction between the components of the application. The components which are source or target of events are not 
specific components. Therefore an event-based style favours the integration of heterogeneous components and services in global applications.

\subsection{Towards Standards for Service-Oriented Technology}

\section{Languages}

Web-Services Description Language (WSDL) The Web-Services Description Language is one of today de facto standard languages to define any kind of services. It is a markup language based on the XML. It is imposed by the industry.

Business Process Execution Language (BPEL) The (Web-Service) Business Process Execution Language ${ }^{2}$ is an orchestration language for web-services; it is used to describe the interactions between web-services. BPEL can be simply viewed as a language to compose services at abstract or execution levels.

\section{Protocols}

Simple Object Access Protocol (SOAP) The Simple Object Access Protocol is an XML-based protocol to exchange information (or messages) in a distributed environment. It can be used in combination with a variety of other (Internet) protocols such as the HTTP protocol. It is also a protocol from the W3C consortium. Technically, the SOAP consists of three layers:

- an external layer that defines a framework for describing message contents and how to process them,

- a layer dedicated to data encoding; it describes a set of rules for expressing instances of application data, and

- a layer dedicated to the representation convention for remote procedure calls and responses.

WSDL is used to describe services based on the SOAP.

Universal Description, Discovery and Integration (UDDI) The Universal Description, Discovery and Integration (UDDI) is a protocol over the HTTP protocol used to describe, locate and discover services.

Web-Services conform to these standards (WSDL, SOAP, UDDI).

${ }^{2}$ www-128.ibm.com/developerworks/library/specification/ws-bpel/ 


\subsection{Service-Oriented Approach and Architecture}

An architecture involving web-services uses three entities and four relationships between them. The involved entities are:

- a service (developer/provider),

- a service registry or server (service library),

- a client.

The relationship are:

- Service Registration, a service is registered on a server (service registry) ; the XML and HTTP protocol are used for that purpose;

- Service Discovery: a client looks for a service by consulting a service registry which in turn sends back the located service to the client; the Universal Description, Discovery Integration (UDDI) protocol is used for this purpose.

- Service Binding. A client is (dynamically) bound to the discovered service. Here, the Web-Service Description Language (WSDL) is used.

- Service Invocation. A client invokes a service to which it is previously bounded. The offered service interacts with its client using the SOAP protocol.

Service Oriented Architecture (SOA), originated from SUN company in the 90's. It is an architectural style to build applications that use the services available on the Internet. The principle is that the services are loosely coupled since they are not originally known but discovered and bind upon demand; by the way, the components of an application are also loosely coupled since the services to link them are used upon demand. The SOA promotes the find, bind, use (services) method. It favours the integration of heterogeneous distributed systems. More details on SOA can be found in 12,11.

\section{Formal Analysis of Component/Service-Based Systems}

In the previous section we have considered the technologies of services as imposed by industries. Note that, considering problems to be solved, industrial time and delays are different from academic ones. Industrial constraints are also different; they often have to produce solutions with restricted delays.

In this section, we consider the academic point of view with respect of software development features, methods and constraints. From this point of view, some stated properties need to be verified for software entities and applications. Verification is still a hard activity that consumes a lot of time and resources (and expertise on formal methods and tools).

Ongoing research efforts in the context of service-oriented applications is for example the Sensoria FET European project 13]. Related works and results on formalisation and semantics of services, reasoning on web-services, and their coordination can be found in $[17,13,17,1,14,96$. 


\subsection{Properties to be verified}

First of all software service construction requires formal specifications in order to permit formal analysis. Expressive specification languages with formal semantics and tools are needed.

The range of properties to be verified is very wide. To cite a few:

- availability of service (reliability of servers),

- functional properties of services,

- reachability properties related to a given service or a functionality,

- side-effect functional properties, for example a called service does not take the control forever,

- correct interaction between a service an its clients; for example, the client is not blocked due to bad interaction with a requested service,

- correct interaction between the services of an application,

- heterogeneity and interoperability questions; services may come from various sources and have different semantic models; how do they interact,

- correctness of message exchange protocols,

- preservation of time-constraints

$-\cdots$

There are a real need for component/service models to face specification and verification aspects.

\subsection{The Kmelia Model Proposal}

We are experimenting on an abstract and formal component model named Kmelia. It is a multi-service component model formally defined [5.2]. In the Kmelia model a component has an interface made of provided services and required services. Services are used as composition units. The provided services have an interface made not only with usage information related to the signatures but also with pre and post conditions in order to ensure at least formal analysis. One strong idea of the model is that the development of component and their services may follow a formal construction (from abstract model to concrete code) in order to enable property verification at different layers.

Service Specification A Kmelia service has the following shape:

SERVICE $<$ serviceName(parameters) $>$ INTERFACE

$<$ dependencies with other (sub)services $>$ PROPERTIES $<$ specific property references $>$ PRE $<$ a predicate $>$ POST $<$ a predicate $>$ BEHAVIOUR

$<$ An extended LTS, with initial and final states $>$ END 
A service $s$ of a component $C$ is defined with an interface $I_{s}$ and a (dynamic) behaviour $\mathcal{B}_{s}:\left\langle I_{s}, \mathcal{B}_{s}\right\rangle$. Usually a required service does not have the same level of detail as a provided service since a part of these details is already in the (provided) service that calls it.

The interface $I_{s}$ of a service $s$ is defined by a 5 -tuple $\left\langle\sigma, P, Q, V_{s}, S_{s}\right\rangle$ where $\sigma$ is the service signature (name, arguments, result),

$P$ is a precondition, $Q$ is a postcondition, $V_{s}$ is a set of local declarations and the service dependency $S_{s}$ is a 4 -tuple $S_{s}=\left\langle s u b_{s}, c a l_{s}, r e q_{s}\right.$, int $\left._{s}\right\rangle$ of disjoint sets where $s u b_{s}\left(\right.$ resp. $\left.c a l_{s}, r e q_{s}, i n t_{s}\right)$ contains the provided services names (resp. the services required from the caller, the services required from any component, the internal services) in the $s$ scope. Using a required service $r$ in $c a l_{p}$ of a service $p$ (as opposed to a component interface) implies $r$ to be provided by the component which calls $p$.

Using a provided service $p$ in the $s u b_{r}$ of a service $r$ but not in the component interface, means that $p$ is accessible only during an interaction with $r$.

The behaviour $\mathcal{B}_{s}$ of a service $s$ is an extended labelled transition system (eLTS) defined by a 6 -tuple $\left\langle S, L, \delta, \Phi, S_{0}, S_{F}\right\rangle$ with $S$ the set of the states of $s$; $L$ is the set of transition labels and $\delta$ is the transition relation $(\delta \in S \times L \rightarrow S)$. $S_{0}$ is the initial state $\left(S_{0} \in S\right), S_{F}$ is the finite set of final states $\left(S_{F} \subseteq S\right), \Phi$ is a state annotation function $\left(\Phi \in S \rightarrow s u b_{s}\right)$. An eLTS is obtained when we allow nested states and transitions. This provides a means to reduce the LTS size and a flexible description with optional behaviours which take the form of sub-service names annotating some states.

Transitions: The elements ( $(s s, l a b e l), t s)$ of $\delta$ have the concrete Kmelia syntax ss--label-->ts where the labels are (possibly guarded) combinations of actions: [guard] action*. The actions may be elementary actions or communication actions. An elementary action (an assignment for example) does not involve other services; it does not use a communication channel. A communication action is either a service call/response or a message communication. Therefore communications are matching pairs: send message(!)-receive message(?), call service(!!)-wait service start(??), emit service result(!!)-wait service result(??). The Kmelia syntax of a communication action (inspired by the Hoare's CSP) is: channel(!|?|!!|??) message (param*).

Channels: A communication channel is established between the interacting services when assembling components. A channel defines a context for the communication actions. At the moment one writes a behaviour, one does not know which components will communicate, but one has to know which channels will be used. A channel is usually named after the required service that represents the context. The placeholder keyword CALLER is a special channel that stands for the channel opened for a service call. From the point of view of a provided service $p$, CALLER is the channel that is open when $p$ is called. From the point of view of the service that calls $p$, this channel is named after one of its required service, which is probably named $p$. The placeholder keyword SELF is a special channel that stands for the channel opened for an internal service call. In this case, the required service is also the provided service. 


\section{Research Issues for Service-based Systems}

\subsection{The Basic Context}

The fundamental context of component or service-based system is that of distributed, asynchronous models. In an asynchronous model a set of processes cooperate to perform a task by exchanging messages over communication support. In some extent the context is that of distributed system with some specificities due to services such as volatility, discovery and dynamic binding. Service-based may be studied as distributed ones [8].

Correctness of component or service-based systems should be studied in this context. The good news are that distributed systems have been well researched, the bad news are that there are still challenging problems that are now exacerbated by the popularisation of web-services and applications.

Numerous works have embraced formal verification of concurrent (distributed) systems 10,30,29,20. The work in [19] focus for example on hardware but with asynchronous mode. In 26,27] the authors use the $\pi$-calculi to reason about services and systems. These are works that can support rigorous analysis of service-oriented systems.

\subsection{Service Construction}

First of all, as a service is a software entity that implements a given functionality, its construction should follows the development lifecycle of any software entity. It may be constructed by refinement from an abstract and formal model. However the specificities of distributed environment and particularly those of services may be taken into consideration: An event-based modelling for example is well-adapted to asynchronous systems. According to service features, the good abstraction level that favours reuse, the appropriate interface for service description and the specific characteristics of the current service should be the parameters of the service construction.

The extension of service standard languages such as WSDL and BPEL is proposed. WSDL may integrate property description features in order to embed the properties inside the service interfaces. The extension may be good at addressing required working conditions description, functional and non-functional properties description and, interaction properties to make them easier.

According to BPEL, the needed extensions may enable an easy checking of composition after extracting/discovering the interaction features. Also an eventbased interaction may facilitate loose composition. There is a need of expressive formally defined languages an tools.

\subsection{Service Composition}

As service-based systems are basically distributed systems, the classical problems studied in the scope of distributed systems should be considered again. The composition of services to build larger applications may cause deadlocks, access conflict to shared resources, race conditions, starvation, etc. 
Moreover, as services should be discovered before being used, planning the composition of services is a challenging question. To find the right services to be composed is a challenging issue that raises again the question of specification matching. Indeed one has to search for a service (which is unknown) with given criteria. The (partial) specification of the needed service is one of these criteria. The desired properties of the searched service are also candidates for the searching criteria. The searching activities are then becoming very tedious. Today searching systems are based on a database of published services which are known and explored by clients which retrieve the needed services.

Correctness of service-based systems requires the proof of global properties by considering hypothesis on the future services that will be found and bound. That means, an analysis of a global system may be performed by considering assumptions on what the required services will be. Therefore, it remains to check that the concrete services satisfy the assumptions.

As far as service composition is concerned, the interconnection between services may be loose since service-based systems have dynamically evolving architecture; this can be modelled and analysed using event-based approaches 3 , : 1 .

The extension of the languages like BPEL is a pragmatic way to undertake the process of improving service composition. An extension enabling one to check the ordering of events and also the temporal properties of service interaction is of real interest.

\subsection{Service Certification}

In order to ensure the quality of a published service which is devoted to be used in building quality software, the certification of the service quality is required. This may be achieved with respect to desired, functional and non-functional properties. A service may be certified as fulfilling given properties. That means the service implementation satisfies its formal specification that states its properties. This is the standard correctness issue required for software entities or applications. However for the convenience of service context, we propose that the services embed their properties so that they can be verified by a third party entities, checked or considered for the integration of the services in other environments.

Property-carrying services are therefore a solution towards the insurance of quality.

A service may at least incorporate its functional specification, the properties that it guaranties and the properties that it assumes for a good functioning.

The client of a provided service which carries its properties may be confident to the provided service. Consequently an application that integrates several services can coordinate and manage the properties required by ones and provided by others. From this setting, reasoning on the entire system my be undertaken. It is not straightforward to establish the correctness in this context of the composition of asynchronous (sub)systems. Works on Assume/Guarantee [31, 22, 18, 16] may be beneficially reused in the component or service-based systems. 
A service should also be certified by a certifier according to the features it has declared. That means the certifier checks the properties embedded in the service and in case of success certifies the service. But the certifier should in turn be a sure one. This matter may be managed by considering well-known provers, efficient proof-checkers and specialised proof centers with experts that perform the proof on demand for the certification purpose. Therefore only the certificates coming from the adopted proof centers may be the confident ones. We develop this idea of property-carrying services from the works on Proof-Carrying Code originally elaborated by Necula [21].

\subsection{Service-based Application and Maintenance}

Service registries may change; services also may change due to upgrades and evolution. Therefore clients of registries may be aware of the modifications and should be able to discover incompatibilities if there are any. How one can be sure that a new version of a service is still correct with its initial objectives. Here again, the certification approach may be used. A service of a system may be replaced by another one without breaking the initial objectives of the system. The formal analysis with respect to hypothesis on services should greatly help in doing this. Service registries should be efficient and adaptable to any form of service descriptions. They should offer the best retrieval features and reasonable response time. Results from database management systems should be exploited.

An important research direction is that of substituability between services or components. A service could be replaced by another one without breaking the chain of functionalities. The service binding should be elaborated to permit such changes. Again, services equipped with properties and certificates will favour this practice.

\subsection{Interoperability of Service-based Systems}

Abstraction is a cornerstone for interoperability. When various systems are based on the same abstract view of the used entities and use predefined rules to exchange and reason about entities of interest, ensuring interoperability becomes easier. Consider for example the well-known Open System Interconnection model of the ISO; it is the reference model that ensures the interoperability between network protocols, operating systems and applications. Rules are defined at different levels of the model, and standards are defined and respected by developers of devices, protocols and software. Few examples are the widely used Transport Control Protocol (TCP), Simple Mail Transfer Protocol (SMTP) or Hyper Text Transfer Protocol (HTTP).

The notions of services and service servers are already present there. Each network service is clearly and uniformly identified by a number that is used by all its clients. When a server is active, its clients requests and accesses the desired services by using the predefined usage manual of this service.

According to service-based systems, exploiting the know-how in the networking, the experiment, methods and techniques of programming distributed system are the main ingredients to ensure quality. Practically, a multi-layered eventbased design and development approach is recommended. 


\subsection{Reliability}

Correctness of services as software entities are not sufficient for reliability. For a service-based system to be reliable, its environment should be taken into account: global correctness, i.e. system level correctness should be considered. The availability of network level services is then an important parameter of the reliability. Permanent availability and good functioning of all the components of the global system is required; obviously this cannot be stated and systematically proved but assumptions may be made to ensure the acceptable reliability according to the considered application. For example, failures of some components may be acceptable, provided that the global system continues to offer a part of its functionalities or to offer its functionalities with more delays.

How to check or detect failures of components/services of the system at hand? When a fixed number of interacting entities is considered, failure can be detected by considering the monitoring of the entities. In case of service-based systems which evolve dynamically as entities are found and bound and unbound, the monitoring is not straightforward. Hence a challenging issue.

Works on dynamically evolving systems, considering group membership and distributed middle-ware systems may be beneficially used here. Ensemble [7, [6] for example is a well-researched system that can be used for service-based systems.

The QuickSilver[ [23] system is also a candidate to ensuring or studying reliability of web-service based systems.

We also advocate for a server of service servers in order to facilitate access and updates. Indeed for a client of services, instead of looking for several service registries for example, it may always contact only one server of the registries which in turn redirects the requests or accesses to the right registries. It will be the role of the server of registries to manage and maintain up-to-date the information concerning the registries. This approach is more flexible and efficient than the former where the clients should know and maintain their information about several registries.

\subsection{Security}

Several problems and solutions have been studied with respect to Internet network applications. As service-based systems is based on this support, the same problems impact these systems and the adopted solutions are also available. Service registries need specific care as they should be continuously available. Technically a cluster of registries may fulfill the objectives. For example, false service registries may be avoided by considering friend service registries. Inaccurate service publishing may be avoided by considering well authenticated service publishers.

\section{Concluding Remarks}

The dissemination of software services through information systems and applications require now and quickly components and services of high quality. Providing

\footnotetext{
${ }^{3}$ QuickSilver Scalable Multicast project website at Cornell: www.cs.cornell.edu/projects/quicksilver/QSM/
} 
verifiable correct services is one solution but it is only partial; more generally, the reliability of component or service-based systems is the true challenge. It goes beyond the components and services correctness; it deals with the environment of the used services, components and developed applications. Layers of properties and correctness proofs are required (services, service registries, components, client applications, certifiers, etc). To follow the objectives of correctness and reliability, it is worth considering the integration of correctness threats in the design and the development of the building blocks which are services. Current languages and techniques can be extended in this direction and they may also exploit existing results on formal analysis of concurrent distributed systems. We have indicated some research issues and work directions. The magnitude of the task is important. But there are already a considerable amount of foundation works that can be beneficially exploited. Results in the field of distributed asynchronous systems modelling and verification are of main interest. Works on property-carrying services certification will provide interesting assessments according to quality. However, efficient property verification techniques, tools and verification centers will be a major part of the effort. Language expressiveness and appropriate modelling techniques, based on specific domains, will help to manage verification complexity, by defining repeatable proof scenario that will accelerate verification and diminish analysis and development cost. Last, efficient simulation environments will be of great help in tuning service construction and service-based applications construction.

\section{References}

1. J. Abreu, L. Bocchi, J. L. Fiadeiro, and A. Lopes. Specifying and Composing Interaction Protocols for Service-oriented System Modelling. In J. Derrick and J. Vain, editors, Formal Methods for Networked and Distributed Systems, volume 4574 of Lecture Notes in Computer Science, pages 358-373. Springer-Verlag, 2007.

2. Pascal André, Gilles Ardourel, and Christian Attiogbé. Composing Components with Shared Services in the Kmelia Model. In 7th International Symposium on Software Composition, volume 4954 of LNCS, 2008.

3. Christian Attiogbé. Multi-process Systems Analysis using Event B: Application to Group Communication Systems. In Z. Liu and J. He, editors, ICFEM'2006, volume 4260 of $L N C S$, pages 660-677, 2006.

4. Christian Attiogbé. Event-Based Approach to Modeling Dynamic Architecture: Application to Mobile Adhoc Network. In T. Margaria and B. Steffen, editors, ISOLA'2008, volume 17 of CCIS, pages 769-781, 2008.

5. Christian Attiogbé, Pascal André, and Gilles Ardourel. Checking Component Composability. In 5th International Symposium on Software Composition, volume 4089 of $L N C S$, pages 18-33, 2006.

6. Kenneth Birman, Robert Hillman, and Stefan Pleisch. Building Network-centric Military Applications over Service Oriented Architectures. In SPIE Defense and Security Symposium 2005, 2005.

7. Kenneth P. Birman, Jie Chen, Kenneth M. Hopkinson, Robert J. Thomas, James S. Thorp, Robbert van Renesse, and Werner Vogels. Overcoming Communications Challenges in Software for Monitoring and Controlling Power Systems. In Proceedings of the IEEE, volume 9, 2005.

8. K.P. Birman. Like it or not, Web Services are Distributed Objects! Comm. of the $A C M$, Viewpoints Column, 2004. 
9. L. Bocchi, Y. Hong, A. Lopes, and J. Fiadeiro. From BPEL to SRML: a Formal Transformational Approach. In M. Dumas and R. Heckel, editors, Web Services and Formal Methods, volume 4937 of LNCS, pages 92-107. Springer-Verlag, 2007.

10. Hana Chockler, Eitan Farchi, Ziv Glazberg, Benny Godlin, Yarden Nir-Buchbinder, and Ishai Rabinovitz. Formal Verification of Concurrent Software: two Case Studies. In Ur and Farchi [28], pages 11-22.

11. Hugh Taylor Eric Pulier, editor. Understanding Enterprise SOA. Wiley India Pvt. ltd, 2006.

12. T. Erl, editor. Service Oriented Architecture Concepts Technology and Desgin. Prentice-Hall, 2005.

13. J. L. Fiadeiro, A. Lopes, and L. Bocchi. A formal approach to service-oriented architecture. In G. Zavattaro M. Bravetti, M. Nunez, editor, Web Services and Formal Methods, volume 4184 of LNCS, pages 193-213. Springer-Verlag, 2006.

14. J. L. Fiadeiro, A. Lopes, and L. Bocchi. Algebraic Semantics of Service Component Modules. In J. L. Fiadeiro and P. Y. Schobbens, editors, Algebraic Development Techniques, volume 4409 of LNCS, pages 37-55. Springer-Verlag, 2007.

15. José Luiz Fiadeiro. Software Services: Scientific Challenge or Industrial Hype? In Zhiming Liu and Keijiro Araki, editors, ICTAC, volume 3407 of LNCS, pages 1-13. Springer, 2004.

16. D. Giannakopoulou, C.S. Pasareanu, and J.M. Cobleigh. Assume-guarantee Verification of Source Code with Design-level Assumptions. International Conference on Software Engineering, (ICSE 2004), pages 211-220, 2004.

17. J. Abreu and J. Fiadeiro. A Coordination Model for Service-oriented Interactions. In D. Lea and G. Zavattaro, editors, Coordination Languages and Models, volume 5052 of Lecture Notes in Computer Science, pages 1-16. Springer-Verlag, 2008.

18. Mooij, A.J. and Wesselink J. W. A Formal Analysis of a Dynamic Distributed Spanning Tree Algorithm. Technical Report No. 03-16, Technische Universiteit Eindhoven, 2003.

19. Katell Morin-Allory, Laurent Fesquet, and Dominique Borrione. Asynchronous Assertion Monitors for multi-Clock Domain System Verification. Rapid System Prototyping, IEEE International Workshop on, 0:98-102, 2006.

20. Gero Mühl, Ludger Fiege, and Peter R. Pietzuch. Distributed Event-Based Systems. Springer, 2006.

21. G. C. Necula. Proof-Carrying Code. In 24th ACM Symposium on Principles of Programming Languages (POPL'97), 1997.

22. Tobias Nipkow and Leonor Prensa Nieto. Owicki/Gries in Isabelle/HOL. In FASE '99: Proceedings of the Second Internationsl Conference on Fundamental Approaches to Software Engineering, pages 188-203, London, UK, 1999. SpringerVerlag.

23. Krzysztof Ostrowski and Kenneth P. Birman. Scalable Group Communication System for Scalable Trust. In STC'06: Proceedings of the first ACM workshop on Scalable trusted computing, pages 3-6, New York, NY, USA, 2006. ACM.

24. Mike P. Papazoglou, Paolo Traverso, Schahram Dustdar, and Frank Leymann. Service-Oriented Computing: a Research Roadmap. Cooperative Inf. Syst., 17(2):223-255, 2008.

25. Mike P. Papazoglou and Willem-Jan van den Heuvel. Service Oriented Architectures: Approaches, Technologies and Research Issues. VLDB, 16(3):389-415, 2007.

26. Marek Rychlý. Towards Verification of Systems of Asynchronous Concurrent Processes. In Proceedings of 9th International Conference ISIM'06, pages 123-130, 2006 . 
27. Marek Rychlý. Behavioural Modeling of Services: from Service-Oriented Architecture to Component-Based System. In Software Engineering Techniques in Progress, pages 13-27. Wroclaw University of Technology, 2008.

28. Shmuel Ur and Eitan Farchi, editors. Proceedings of the 4th Workshop on Parallel and Distributed Systems: Testing, Analysis, and Debugging, held in conjunction with the ACM SIGSOFT International Symposium on Software Testing and Analysis (ISSTA 2006), PADTAD'2006. ACM, 2006.

29. J.W. Wesselink, J.M.T. Romijn, and A.J Mooij. Assertion-based Proof Checking of Chang-Roberts Leader Election in PVS. In K.S. Namjoshi, T. Yoneda, T. Higashino, and Y. Okamura, editors, (Proceedings 5th International Symposium, ATVA 2007), volume 4762 of Lecture Notes in Computer Science, pages 341-361. Springer-Verlag, 2007.

30. Margaret A. Wojcicki and Paul A. Strooper. A state-of-practice Questionnaire on Verification and Validation for Concurrent Programs. In Ur and Farchi [28], pages $1-10$.

31. Q. Xu and M. Swarup. Compositional Reasoning Using the AssumptionCommitment Paradigm. LNCS, 1536:565-583, 1998. 